\title{
The good, the bad, and the ugly
}

\section{Fred T Bosman}

Published online: 20 December 2014

(C) Springer-Verlag Berlin Heidelberg 2014

In this classical Western movie, the guy nicknamed "the bad" faces a gun and says to its owner who is ready to shoot; "if you miss, you had better miss very well". The Editorial team of Virchows Archiv (VIAR) feels a bit in that position. In working on and for VIAR, we want to have an impact. If our pretention is to be among the top Pathology journals in addition to being the oldest, we need to apply an explicit and coherent strategy. We might be shown wrong in some of the choices made, but at least, we will have worked on implementing a clear vision.

Let us start with the ugly. I am sure that you did not immediately recognize the journal, and I would not be surprised if the new morphology (to stay in pathospeak) of the journal stirs up a bit of discussion. The "old" cover was considered by many of its readers as ugly and not befitting a pathology journal. The flashy colors without a discernable pattern might suggest to nonpathologists that histomorphology remains more of an art than a science. Others liked the colors: unconventional, visually pleasing with a hint of "flower power". Some of you will like the new cover: kind of EURO-blue, befitting for the "European Journal of Pathology" notably with the EURO-yellow lettering of the name of our founding father, more informative and with an image illustrating an item in the issue. Others will comment that the cover will blend in with all the other (pathology) journals with a blue cover and has lost some of its visual appeal. But then, what's the importance of the cover, other than creating a buzz and visual identity? Isn't what is in the journal the most important?

And then the good. We are making good progress on the strategic choices made. The number of review papers has gone up, and overall, these are appreciated. The annual review issue has been very positively received, and only time will tell if the high number of downloads of the papers will translate into loads of citations. The number of submitted original

F. T. Bosman $(\bowtie)$

Institute of Pathology, University Medical Center (CHUV) of

Lausanne, Lausanne, Switzerland

e-mail: Fred.Bosman@chuv.ch manuscripts keeps going up, and with the set number of pages we can print annually, we can be more selective in accepting papers for publication. Some initiatives are still pending or need additional efforts. We still intend to start publishing teaching cases in a quiz-like format. That requires a series of cases relevant in terms of educational value and presented in such a way that reflection is stimulated. To do this properly requires manpower of which we have little. We will keep the number of case reports down, although we continuously receive many case report manuscripts to be almost immediately rejected. Authors should read our aims and scope and author instructions better! Much of the published material is based upon translational studies with potential impact on future practice, which is timely. Writing quality improves, although your editor in chief still spends much time improving on the writing and increasingly requests authors to have manuscripts edited by a professional. Think about this before you submit.

To end with the bad. The impact factor (IF) stayed at the same level (for some) or went down a little bit (for others). Is this really bad? Is the impact factor really important for us? It is not my intention to contribute my personal views to the expanding debate on the virtues (or lack thereof) of bibliographic parameters and notoriously the IF. I cannot hide the fact that the visibility of the journal is in part determined by its IF. We have to realize that our IF relies heavily on citations of review papers, which are a reflection of editorial policy and much less on the intrinsic value of the published original material. Lots of mediocre material are published in high impact journals and might be regarded as impressive in terms of bibliographic parameters, even though maybe poorly read and not much cited. Assessing the value of a paper requires more than applying a parameter based upon what the journal publishes in general. For us editors, the IF remains of some importance; while for you, author of an original manuscript, it is the actual citation frequency of your paper that is much more important. So, continue to submit your (good) papers to VIAR. We will continue to solicit and publish more reviews; we know you like them and yes, they do push up the IF. 